\title{
The Total Network Capacity of Wireless Mesh Networks for IoT Applications
}

\author{
https://doi.org/10.3991/ijim.v14i08.12697
}

\author{
El Miloud Ar-Reyouchi $\left.{ }^{(}\right)$ \\ Abdelmalek Essaadi University and SNRT Tetouan, Rabat, Morocco \\ e.arreyouchi@m.ieice.org \\ Yousra Lamrani, Imane Benchaib, Kamal Ghoumid \\ ENSAO, Mohammed I University, Oujda, Morocco \\ Salma Rattal \\ FSTM Hassan II University, Casablanca, Morocco
}

\begin{abstract}
Computing and measuring the total capacity of a data network are a remarkably difficult problem. These metrics are directly linked to the available bandwidth to each wireless internet of things (IoT) device of the network. In this paper, the authors study the performance metrics associated with capacity traffic in multi-hop wireless mesh networks (WMNs). It is dedicated to Internet access, assuming a time division multiple access (TDMA). They focus simultaneously on three key operating metrics, the total network capacity (TNC), total application network capacity (TANC), and the Average message time (AMS). They also analyze how parameters such as forward error correction (FEC) and acknowledgments (ACK) affect the overall network capacity under different operating conditions. Theoretical network capacity for WMNs, in this paper, is explored to draw attention to the number of open research issues
\end{abstract}

Keywords - Wireless mesh network, network capacity, average message size, routing, network topology.

\section{$1 \quad$ Introduction}

WMNs are emerging as the more lucrative technology for determining wideband connectivity [1]. However, for ensuring the smooth operation and meeting the requirements of the applications, the applications require higher throughput; consequently, WMNs need a higher capacity for innovating solutions. The network capacity of a wireless mesh network (WMN) [2] implemented in the internet of things (IoT) is one of the significant criteria for quality of service.

With rapidly expanding numbers of WMNs [3] available for IoT [4] applications, developers need to understand how these networks differ concerning the use cases and expected performance. 
Many parameters, such as error-Correcting Codes (ECC), message size transmitted, network topology, traffic pattern, network node density, number of channels used for each node, transmission power level, and node mobility, can influence the capacity of WMNs. The testing focused on device behavior and impact of AMS, FEC, and ACK [5] on network capacity, total IP network capacity, and total application network capacity. The network capacity can be enhanced by growing the number of gateways if they are sufficiently spaced from each other [6]. In effect, the network capacity is constrained by the activity inside a bottleneck zone around the gateway [6,7].

A significant surge in demand for total IP/application network capacity is invading the industrial internet of things (IIoT) [8] caused by an ascendant demand from IoT devices, and consumers with an exceptional and high ambition for real-time access to massive amounts of data services. Confront this insatiable consumer traffic demand; operators are obliged to enlarge network capacity. In the context of WMNs, network capacity (in bytes per second) can be considered as the intricate measurement of the maximum quantity of data that may be conveyed between network places over a network path. Because of the number of intertwined measurement variables and complicated scenarios, the actual network capacity metric is rarely found with a precise measure. In [9], the authors address the problem of calculating the transport capacity of WMNs destined to the internet. Capacity is also known as data rate or as throughput. Latterly, network coding technique has been used in wireless networks communication for improving network capacity, and it can also optimize the broadcast Strategy [10].

Network capacity planning is a critical approach of conveyable network architecture planning to guarantee an efficiency network has the increased capacity to meet future needs [11]. Analyses of the transmission capacity of multi-hop, wireless mesh networks regularly connect bounds on the maximal achievable data rate to spatial reuse constraints and MAC-layer impacts. In WMNs, all IoT nodes employ the identical physical channel (such as IEEE 802.11 [12] based ad hoc LANs). The total network capacity is certainly conditional on the coverage area of each wireless IoT node. Since a packet, transmission by a node efficiently precludes any transmissions, happening at about the same time, by neighboring IoT nodes (within its interference range).

Managing network capacity, to optimize performance depends on several essential parameters, namely: Rate at which handsets enter and leave a covered cell site area, average message size [13], average hops per path, modulation (type and rate), subscriber services, FEC and ACK, its parameters can also affect Trip Time Metric in multi-hop WMN as in [14]. Also, the number of nodes affects network capacity as in dense wireless sensor networks (DWSNs), which is one of the fundamental parameters such as in [15].

The IoT carriers are forced to grow network capacity to accommodate exploiters of IoT requests for high-bandwidth services. Nowadays, network capacity is required to handle increased IoT devices and supplementary services. For a similar reason, the total achievable network capacity is also a function of the IoT nodes density, which implicitly determines the average number of one-hop neighbors. In the paper [16], the authors analyze the capacity of wireless networks. With randomly located nodes con- 
sidering two types of networks, arbitrary, and random networks. The authors of [17] expect the total capacity of wireless ad hoc networks, mainly; it studies 802.11 MAC interactions with ad hoc forwarding, their effect on network capacity

In this work, the authors analyze the network capacity of WMNs. It is assumed that each node is located in the middle of the region of the area, and it can transmit at bits per second over a shared wireless channel. Packets are sent from station to station in a multi-hop mode until they reach their single final destination (remote station). They can be buffered at intermediate stations while awaiting transmission. To simplify the tasks of the nodes, the medium access mode to be used is a TDMA, as applied in [13]. TDMA is a successful method for avoiding packet collisions. However, time slot distribution must be realized to use TDMA.

The paper presents wireless mesh networks and the effect of FEC and acknowledgments (ACKs) in IoT networks. It analyses and discusses network capacity, gives an overview of the system model, and presents contributions on some measurements and numerical results obtained for the described model.

\subsection{Wireless mesh network}

Wireless mesh network (WMN) is a wireless local area network (WLAN) using one or more decentralized IoT devices connection, include complete mesh topology or partial mesh topology. In a complete mesh topology, each network IoT node is connected directly to each of the other IoT nodes. In a partial mesh topology, some IoT nodes are connected to all the others, but others are only connected to those IoT nodes with which they interchange the most data. WMN is a promising wireless communication technology for various applications [18] to comply with specific requirements and uses.

In WMN, all IoT devices can access each other randomly and spontaneously, and each network IoT node can forward data to the next IoT node. The networking infrastructure is decentralized and simplified because each IoT node need only transmit as far as the next IoT node. Wireless mesh networking could permit users living in remote areas operating in rural neighborhoods to connect their networks for available IoT connectivity.

In wireless mesh-type networks, all IoT devices can access each other arbitrarily and spontaneously. WMNs can also accomplice polling or report-by-exception applications.

Each link between IoT nodes (and) could be noisy, so the information in the packets also needs to be encoded to verify and correct the errors. In this paper we analyze FEC, and ACK approaches, a class of time-diversity techniques, in the context of IoT network at the link and transport layers

\subsection{Effect of FEC and acknowledgments (ACKs) in IoT network}

FEC (Forward Error Correction) is an instrumental technique to understate IoT wireless channel weakness. The transmitter subjoins some redundant data into its messages. This redundancy permits the receiver to detect and correct the erroneous 
packet. The improvement comes at the expense of the data transfer rate concerning each IoT devices.

$R_{\text {data }}=m \times r_{F E C}$, where $R_{\text {data }}$ is the IoT device data rate, $m$ is the modulation rate, and $r_{F E C}=$ FEC ratio.

The above formula shows that the lower the FEC ratio, the better the ability of error correction and the lower the IoT device data rate.

ACK is a setting, which requires additional bandwidth to repeat corrupted frames. To announce that the packet was received correctly, each transmitted packet has to be acknowledged by the receiving IoT device, using the very short service packet (ACK). If ACK is not received, the IoT device will retransmit the packet depending on its set of new attempts. The authors in [19] analyze the effects of packet forwarding in Mesh Network on transmission performance.

When the IoT device has successfully received the transmitted packet, it no needs to reconstruct any dropped, lost, and missing packets at the receiver. Consequently, there is no requirement to receive ACK from the receiving IoT device, i.e., the packet is transmitted only once, and it is not re-iterated. It is noted that the acknowledgment/retransmission scheme is an integrated part of the radio protocol. It can work individually and separately of any new attempts at higher protocol levels (e.g., TCP or IoT devices application protocol).

\section{Network Capacity}

Starting from a given state of the network in which it is assumed that only TCP traffic is being processed. The network capacity is defined as the maximum TCP throughput that all IoT devices in their network could carry set. If each of them were to download as much as they can. Consider a WMN using a single radio channel shared among all routers that are assumed static. We distinguish the network capacity, defined as the maximum capacity of a link or network path to convey data from source (SCADA center node) in the network to the destination (remote station) via intermediate nodes.

In the context of the Internet of Things (IoT), a better network-wide capacity is necessary for providing a better quality of service to a more significant number of users.

In this paper, the TNC in bytes per second (includes IP packet overhead) is defined as the resulting number mentions to the maximum number in the ideally designed IoT node network. Notwithstanding, that figure can be solely completed when there is a considerable portion of the communication load among the IoT nodes (remotes) themselves.

When all control messages have ultimately to reach the SCADA center station over the same links employing the same radio-channel, any calculation of TNC loses its sense. This problem can be eliminated by adding additional channels or bypass dominant IoT devices using more radio hops. Usually, every network of devices using narrowband radios demands a capacity-aware layout. 
Consider the network in Fig.1, which consists of three $(n=3)$ wireless routers coverages areas. Router 1 sends its packets to router 2 , and, thus, the link $e_{1,2}$ has to be able to forward traffic. Router 2 starts forwarding its packets to router 3 via the link $e_{2,3}$. Router 1 can exchange data with Router 2 . Router 3 can also exchange information with Router 2. However, Router 1 and 3 cannot communicate with each other, but their signals can interfere at Router 2.

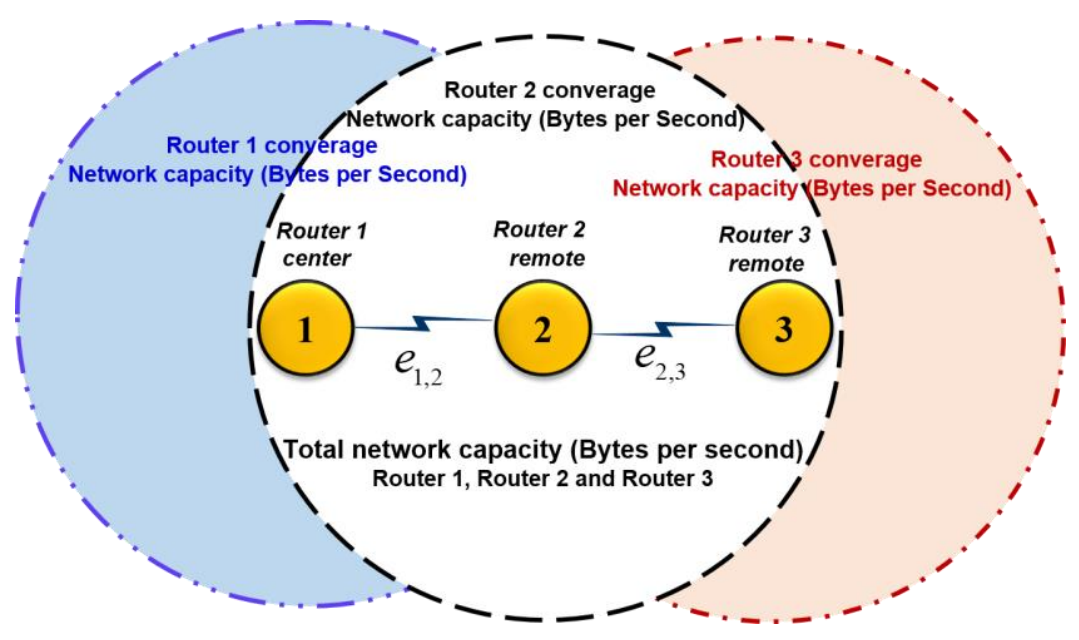

Fig. 1. The basic concept of the Total Network Capacity in wireless IoT devices.

Digital radio has developed ways in which more than one information conversation of the data center can be accommodated (multiplexed) inside the same physical radio channel. For achieving this, the TDMA method is used. It must be noted that in that case, TNC is dependent on the transmission range of each IoT node. So that the collisions cannot be excluded entirely; hence, a collision-solving system must be a complete part of the protocol in the radio channel.

Whenever the traffic increases over a specific limit, the number of collisions augments considerably, decreasing the instant network capacity well below normal situation.

\section{System Model}

In this paper, the fixed infrastructure of the WMN is represented by an acyclic directed graph $G=(V, E)$ with an IoT node-set $V=\left\{v_{1}, \ldots, v_{n}\right\}$ representing mesh points and an edge set $E$. The directed edge connecting the IoT node $v_{i}$ to the IoT node ${ }^{v_{j}}$ is denoted by $e_{i, j}$. 
It is assumed that all the IoT nodes are synchronized. Each edge $e_{i, j}$ has a capacity $C_{i, j}$ (bits/sec), meaning that a packet of $L$ bits is transmitted in at least $L / C_{i, j}$ seconds. Consider that, for each link $e_{i, j}$, the maximum transmission delay of a packet of $L$ bits is known and equal to $L / C_{i, j}+T_{i, j}$, where $T_{i, j}$ is the service delay over the links $e_{i, j}$.

Fig. 2 shows the SCADA center and a chain of $n$ IoT nodes, which respectively generates and forward traffic to the remote station (RS).

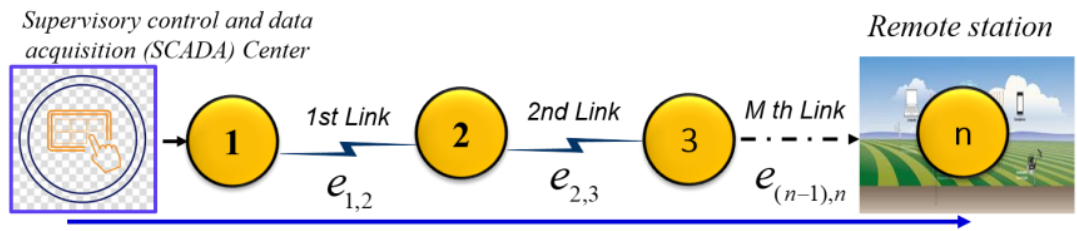

Total Network Capacity

Fig. 2. Total Network Capacity with $M$ links, a chain of $n$ nodes generating and forwarding data to a remote station.

SCADA center sends control information to all IoT nodes. SCADA application typically uses a specific address for such control information. Along the paths of the highway system, one node is chosen per edge, which acts as a relay for the following node. The receiving IoT node 2 (directly connected to the IoT node 1 (one radio hop), converts such message to a customized IP broadcast and sends it to IoT node 3 over one IoT node 2 (two radio hops) respectively to all node's units within the network. Each link in the chain is constrained to send only when the other links in its vicinity are inactive. An objective to expand the system in WMNs is to stretch the coverage range of wireless IoT networks without resorting to the sacrifice of the channel capacity. Another aim is to provide non-line-of-sight (NLOS) connectivity among the IoT devices without direct line-of-sight (LOS) links. To meet these needs, the multi-hop paths are required [20]. Wireless mesh networking has become ideal for IoT, for smart car parking mobile application[21], and it can be incorporated with different network capacities such as IEEE 802.11, 802.16, 802.15, etc.

Assume that each IoT node receives traffic to be forwarded to the RS and that each IoT node can only receive packets from its immediate neighbors. The traffic that has to be forwarded by each link is computed. It is clear from the picture that nodes closer to the RS have to forward more traffic than nodes farther. The RS is connected to the SCADA center through $n$ IoT nodes, with the last one is considered as a remote station.

The packet must be completely received before being forwarded to the next IoT node. Consider $D$ as a total delay for sending $N$ packets crossing $n$ nodes over $M$ links (pipelining delay) that is given in the following form [22]:

$\mathrm{D}=$ Propagation delay + Transmission Delay + Pipelining delay 


$$
D=D_{p}+N * D_{T P}+(M-1) * D_{T P}
$$

Where $D_{T P}=$ transmission time of the packet. Each packet contains $L$ bits of data and a header of size $H$ bits with total packet size $L+H$ bits.

$D_{p}=\frac{d_{\text {total }}}{V_{p}}$, with $d_{\text {total }}$ is the total distance between SCADA center and remote station and $V_{p}$ is propagation speed over the specific medium.

The transmission of a message (control information) of $S$ bits needs $S / L$ packets; therefore, the time to transmit the message over M links is

$$
D=\frac{d_{\text {total }}}{V_{p}}+\frac{S}{L}\left(\frac{L+H}{R}\right)+(M-1)\left(\frac{L+H}{R}\right)
$$

where $R$ is the data rate.

In this network, there is one source node (SCADA center) and one destination node (remote station). The source node does not have any incoming edges, and a destination node does not have any outgoing edges. SCADA center has a message for monitoring remote station system as in [19].

Consider that the total network capacity $C_{T N C}$ is the relationship between the useful data trafficked, without any headers (IP, TCP, UDP,...), trafficked by all IoT devices and the total time takes them, so:

$$
C_{T N C}=\frac{n \times L}{\sum_{i=1}^{n} T_{i}}
$$

where $n$ is the number of connected IoT devices and $T_{i}$ is the average time, it takes each one to traffic $L$ bytes of payload.

$$
T_{i}=\operatorname{aSlot} \text { Time } \cdot \frac{\left(W_{\min }-1\right)}{2}+T_{\text {data }}+T_{A C K}
$$

where $T_{\text {data }}+T_{A C K}$ is the total time it takes for a node $i$ to download a full packet payload $L$.

Rewriting the equation (3):

$$
C_{T N C}=\frac{1}{\frac{1}{n} \sum_{i=1}^{n} \frac{T_{i}}{L}}
$$

Consider that $C_{i}$ is the capacity that each IoT node obtained. Consider the case where only the one connected to the network which is equivalent to: 


$$
C_{T N C}=\frac{1}{\sum_{i=1}^{n} \frac{1}{n} \frac{1}{C_{i}}}
$$

This last result represents the total network capacity assuming that all connected users are reporting as much as possible.

Total network capacity in bytes per second (includes IP packet overhead) is the resulting number refers to the maximum number in the optimally designed IoT devices network. While total application network capacity is total network capacity in bytes per second, but no IP packet overhead is included.

The following Table 1 lists the most critical measured parameter selected for the used topology.

Table 1. Setting Measurement Parameters.

\begin{tabular}{|l|c|}
\hline \multicolumn{1}{|c|}{ Settings } & Status /Mode /Type/Value \\
\hline Average message size (bytes) & $0-1500(1460)$ \\
\hline Processing time(msec) & 20 \\
\hline Interface speed & ETH TCP/IP \\
\hline Modulation & QAM \\
\hline FEC, ACK & On /Off \\
\hline Nodes number & 9 \\
\hline The output power of each device & 10 watts \\
\hline aSlotTime & $9-20 \mu s$ \\
\hline Wmin & 15 \\
\hline Wmax & 1023 \\
\hline$n$ & 9 \\
\hline
\end{tabular}

Usually, the maximum transmission unit (MTU), $M T U_{\text {ethernet }}=1500=1460+20+20$ bytes corresponding to the payload, IP header, and TCP header respectively, consequently $L$ takes the value of 1460 bytes.

Assuming there are no collisions, the average time of each countdown is $\left(W_{\min }-1\right) / 2$ time slots. For the first transmission, and after each successful transmission, the $\mathrm{W}$ value takes the $W_{\min }$ value. For each retransmission, this value $W$ doubles until it reaches the $W_{\max }$ limit, the moment from which $W$ stops growing. Then the plot discards a certain number of retransmissions. Also, it is assumed that there are only one SCADA center and one remote station in the network. Furthermore, every IoT node is capable of creating, receiving, or transmitting data over a communications channel. The topology illustrated in Fig.2 is respected for the simulation with $n=9$.

\section{$4 \quad$ Results}

This section describes the results of the experiments. The presented measurements show how Total application network capacity (TANC) varies depending on the sever- 
al Message sizes according to the modulation type (QAM). Various measurements are effectuated as a function of different values of AMS, without FEC/ACK or with FEC/ACK, respectively. Each device may support up to 1500 bytes of RF payload. In this case, the payload bitrate curve shape, depending upon the number of hops, AMS, and $\mathrm{ACK} / \mathrm{FEC}$, is illustrated in the figures that follow.

The Fig. 3 shows the results of TANC in bytes per second, (but no IP packet overhead is included) versus AMS with modulation type QAM.

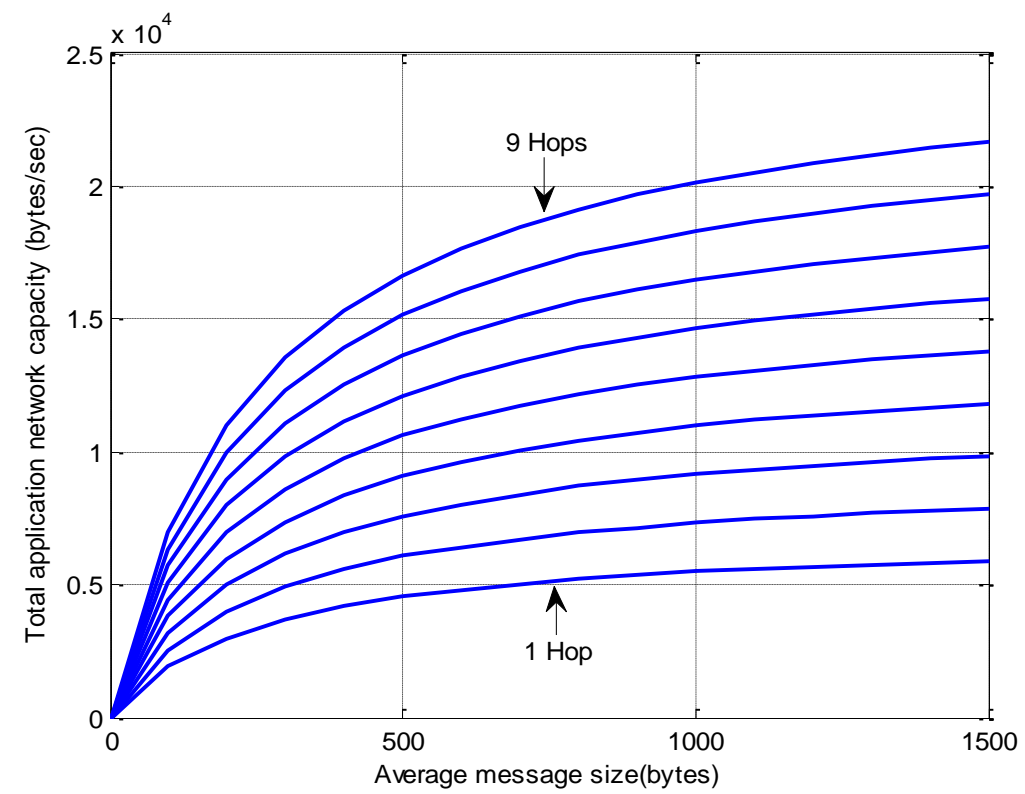

Fig. 3. TANC vs AMS: from $n=1$ hop to $n=9$ hops with identical retransmissions of Rayleigh channels using BPSK modulation Multi-Hop path.

According to the results shown in Fig. 3, it can be observed that the TANC increases considerably for the AMS values increase until 500 bytes, then increases slowly from 500 bytes to 1500 bytes. Additionally, one can see that the number of hops can also promote a significant increase in TANC.

The results illustrated in Fig. 4 show the comparison between Total IP network capacity and Total application network capacity without any correction. 


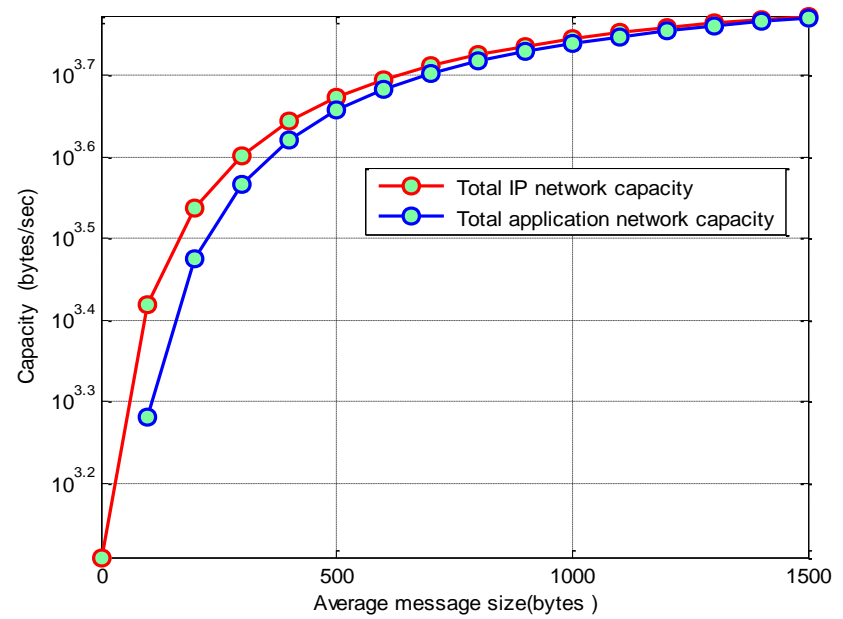

Fig. 4. Total IP network capacity and Total application network capacity without any correction.

It is noted that the total IP network capacity is high relative to the capacity total application network capacity, especially between zero and 600 bytes.

Fig.5 shows the effect of protocols (on network capacity), which can detect and recover the lost packets, either by data redundancy $\mathrm{FEC}=\mathrm{On}$ or by retransmission $\mathrm{ACK}=\mathrm{ON}$.

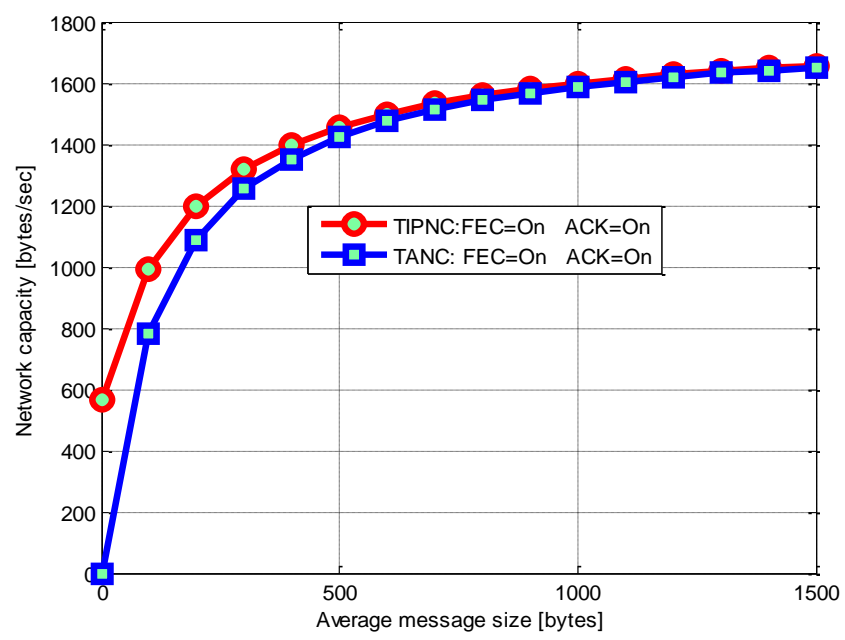

Fig. 5. Total IP network capacity and Total application network capacity without any correction. 
The results in Fig.5 show that FEC, as well as ACK, have a significant effect on the network capacity. Additionally, our results reveal that packet loss recovery techniques promote a further decrease in network capacity.

The scenario is considered in Fig.2: direct transmission between the serial neighbor devices using nine routers. Fig. 6 shows the comparison of Total IP network capacity results obtained for different configurations of FEC and ACK by considering different fixed message sizes.

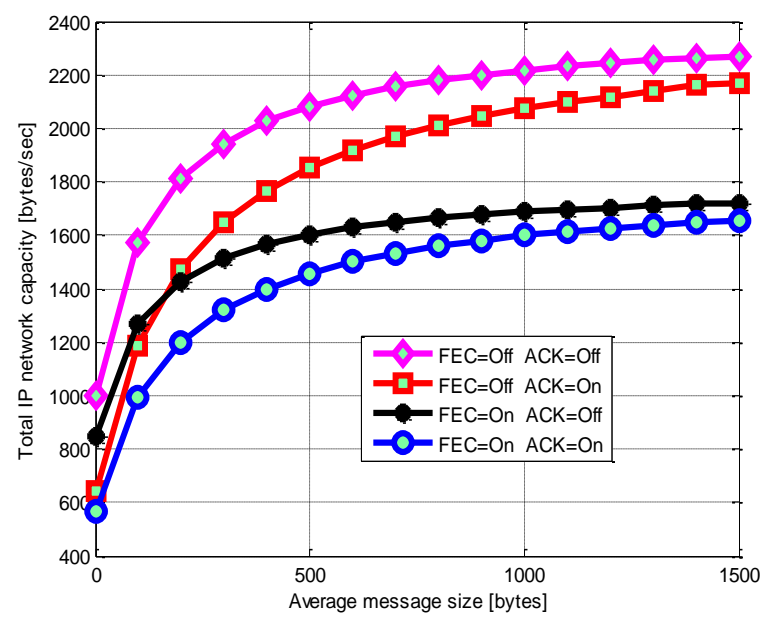

Fig. 6. Total IP network capacity vs. AMS using different configurations of FEC and ACK.

The results in Fig. 6 show that the using different average message sizes (bytes) in wireless networks, without FEC/ACK or with FEC/ACK respectively, has a significant effect on the Total IP network capacity. Additionally, the results reveal that the FEC decreases the total IP network capacity channel more than ACK.

In the same way, Fig.7 shows the comparison of total application network capacity results obtained for different configurations of FEC and ACK. 


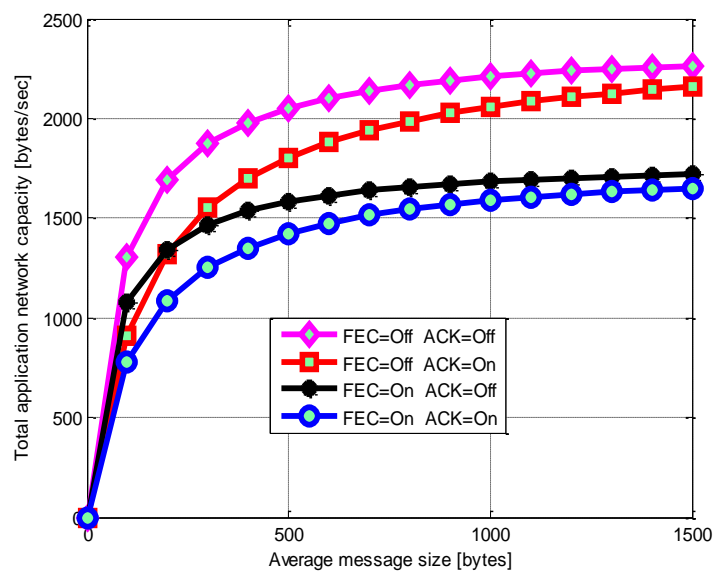

Fig. 7. Total application network capacity vs. AMS using different FEC/ACK configurations

The introduction of ACK increases the Total IP network capacity and total application network capacity more than the presence of FEC. Also, without any correction $(\mathrm{FEC}=\mathrm{ACK}=0)$ only increases capacity but does not improve robustness to link failures, network congestion, and other problems of the wireless network communication.

Moreover, it can be noted that the results illustrate that the IP packet overhead promotes a further decrease in Total application network capacity. The use of FEC and ACK has the disadvantage of low network capacity.

To ensure the conformity of the experimental results, it convenient to compare them with the simulation result, as illustrated in Fig.8.

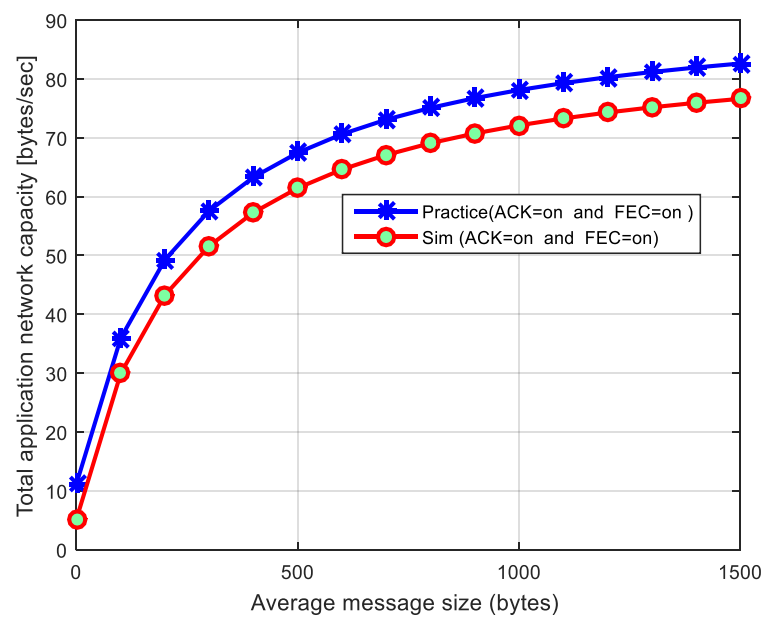

Fig. 8. Comparison between the simulation and measurement results of Total application network capacity vs. AMS using FEC and ACK. 
In the case where ACK and FEC have become operational, the curve illustrated in Fig. 8 shows that the experimental and simulation results are almost the same

\section{Conclusion}

In this paper, the authors focus on the analysis of TNC over a multi-hop, wireless network technologies, designed for the IoT, where all links share the same physical channel. Our analysis shows that it is often difficult to simultaneously improve both the TNC and the Error Correction Code (ECC). Number hops and average message size adversely affects the TNC. They analyze the case in which the noise in a given link is unrelated to the signals traveling over other links. In that case, the authors show, the problems of error correction and capacity network can be separated without limiting the capacity of the network as a whole.

Furthermore, the analysis of the results shows that the Error-correcting codes, such as FEC and ARQ, have two opposite effects on the efficiency of TNC in IoT networks with different lengths. Although ECC dramatically increases the reliability and performance of the overall system, the ECC also makes signals more robust; in contrast, it reduces the total network spare capacity. It also shows that there is a straightforward connection between TNC characteristics and observed packet size FEC and ACK. The more hops per path, the less overlap, and consequently, more capacity left for simultaneous transmissions from different IoT devices. That is the reason for higher capacity with more hops in the network, and it is the subject of the future work using a new performance algorithm.

\section{References}

[1] I.F. Akyildiz, X. Wang, W. Wang. (2005) .Wireless mesh networks: a survey. Computer Networks Journal 47 (4), 445-487. https://doi.org/10.1016/j.comnet.2004.12.001

[2] P. Gupta, P.R. Kumar.(2000). The capacity of wireless networks. IEEE Transactions on Information Theory 46 (2), 388-404.

[3] I. F. Akyildiz and X. Wang.(2005). A Survey on Wireless Mesh Networks. IEEE Communications Magazine, 43(9). S23 - S30. https://doi.org/10.1109/mcom.2005.1509968

[4] Y. Liu, K.-F. Tong, X. Qiu, Y. Liu, X. Ding. (20017). Wireless mesh networks in IoT networks. International Workshop on Electromagnetics: Applications and Student Innovation Competition (iWEM), 183-185. https://doi.org/10.1109/iwem.2017.7968828

[5] El Miloud AR Reyouchi, Y. Chatei Y, K. Ghoumid, A. Lichioui. (2015). The Powerful Combined Effect of Forward Error Correction and Automatic Repeat Request to Improve the Reliability in the Wireless Communications. International conference on computational science and computational intelligence (CSCI), Las Vegas, NV, USA.691696https://doi.org/10.1109/csci.2015.39.

[6] Anis Ouni, Hervé Rivano, Fabrice Valois. (2010). Capacity of wireless mesh networks: determining elements and insensible characters. PlanNet, International Workshop on Planning and Optimization of Wireless Communication Networks (IEEE WCNC2010 Workshop), Sydney, Australia. https://doi.org/10.1109/wcncw.2010.5487652 
[7] C. Gomes, S. Pérennes, and H. Rivano, Bottleneck.(2008). Analysis for routing and call scheduling in multi-hop wireless networks. In 4th IEEE Workshop on Broadband Wireless Access (BWA), New Orleans, US. https://doi.org/10.1109/glocomw.2008.ecp.78

[8] Li Da Xu, Wu He, Shancang Li.(2014). Internet of things in industries: a survey, IEEE Transactions on Industrial Informatics, 10(4), 2233-2243. https://doi.org/10.1109/tii.2014. $\underline{2300753}$

[9] Christelle Caillouet, Stéphane Pérennes, Herve Rivano.(2014). Framework for optimizing the capacity of wireless mesh networks". Computer Communications, 34(13).645-1659. https://doi.org/10.1016/j.comcom.2011.03.002

[10] Muktar Hussaini, Shahrudin Awang Nor, Amran Ahmad. (2012).Optimal Broadcast Strategy-Based Producer Mobility Support Scheme for Named Data Networking. https://doi.org/10.3991/ijim.v13i04.10513

[11] Qian Clara Li, Huaning Niu, Apostolos Tolis, Papathanassiou. (2014). 5G network capacity: key elements and technologies, IEEE Vehicular Technology Magazine 9(1). 71-78 https://doi.org/10.1109/mvt.2013.2295070

[12] IEEE Computer Society LAN MAN Standards Committee.(2012) Wireless LAN Medium Access Control (MAC) and Physical Layer (PHY) Specifications IEEE Std. 802.11-1197. https://doi.org/10.1109/ieeestd.1997.85951

[13] Youssra Chatei, Maria Hammouti, El Miloud Ar-reyouchi, and Kamal Ghoumid. (2017). Downlink and Uplink Message Size affect Round Trip Time Metric in Multi-Hop Wireless Mesh Networks. International Journal of Advanced Computer Science and Applications (IJACSA), 8(3).223-229. https://doi.org/10.14569/ijacsa.2017.080332

[14] Maria Hammouti, El Miloud Ar-reyouchi, Kamal Ghoumid, and Ahmed Lichioui. (2016). Clustering Analysis of Wireless Sensor Network Based on Network Coding with LowDensity Parity-Check. International Journal of Advanced Computer Science and Applications (IJACSA), 7(3).137.143. https://doi.org/10.14569/ijacsa.2016.070319

[15] El Miloud Ar-Reyouchi, Youssra Chatei, Kamal Ghoumid, Maria Hammouti and Bekkay Hajji (2017). Efficient coding techniques algorithm for cluster-heads communication in wireless sensor networks. AEÜ-International Journal of Electronics and Communications. 82. 294-304. https://doi.org/10.1016/j.aeue.2017.08.047

[16] P. Gupta, P.R. Kumar. (2000). The Capacity of Wireless Networks, IEEE Transactions on Information Theory.46 (2). 388 - 404. https://doi.org/10.1109/18.825799

[17] Jinyang Li, Charles Blake, Douglas S.J. De Couto, Hu Imm Lee, Robert Morris. (2001). Capacity of Ad Hoc Wireless Networks. Proceeding MobiCom '01 Proceedings of the 7th annual international conference on Mobile computing and networking, Rome. 61-69. https://doi.org/10.1145/381677.381684

[18] Mesh Networking Forum (2004). Building the business case for implementation of wireless mesh networks. Mesh Networking Forum 2004, San Francisco, CA.

[19] Soumen Kanrar. (2019). Efficient Packet Forwarding in Mesh Network. (2012). International Journal of Interactive Mobile Technologies (iJIM), 6(2), 4750.https://doi.org/10.3991/ijim.v6i2.1991

[20] L. Krishnamurthy, S. Conner, M. Yarvis, J. Chhabra, C. Ellison, C. Brabenac, E. Tsui.(2002). Meeting the demands of the digital home with high-speed multi-hop wireless networks, Intel Technology Journal 6 (4). 57-68.

[21] Aekarat Saeliw, Watcharasuda Hualkasin, Supattra Puttinaovarat, Kanit Khaimook (2019). Smart Car Parking Mobile Application based on RFID and IoT. Journal of Interactive Mobile Technologies (iJIM).13(5),4-14. https://doi.org/10.3991/ijim.v13i05.10096

[22] Imane Maslouhi, El Miloud Ar-reyouchi, Kamal Ghoumid, Kaoutar Baibai. (2018). Analysis of End-to-End Packet Delay for Internet of Things in Wireless Communications. In- 
ternational Journal of Advanced Computer Science and Applications (IJACSA), 9(9), .338-343. https://doi.org/10.14569/ijacsa.2018.090944

\section{Authors}

El Miloud Ar-Reyouchi is a member of the IEEE Broadcast Technology Society, IEEE: New York, NY, US, and member of IEICE, Institute of Electronics, Information and Communication Engineers: Tokyo, JP. He works as regional Manager of the centers of the broadcast TV/radio FM of SNRT Morocco. He is with Abdelmalek Essaadi University, Dep. Telecommunication, and Computer Engineering and with E.T.S Dep. Computer Science and Control, Madrid, Spain. Email: e.arreyouchi@m.ieice.org

Yousra Lamrani holds an engineering degree in Telecommunications and Networks from the National School of Applied Sciences (ENSAO) in Oujda. Currently, she is working on her PhD at the ENSAO (Mohammed Premier University), specializing in Telecommunications Systems at the laboratory of "Signals, Systems and information processing". For her thesis topic, Yousra is studying the performance of network coding in future DVB-T and DVB-S broadcasting standards.

Imane Benchaib holds an engineering degree in 'Electronic systems, Informatics and Networks' from the National School of Applied Sciences (ENSAO) in Oujda. Currently, she is working on her PhD at the ENSAO (Mohammed Premier University), specializing in Communication Networks at the laboratory of "Signals, Systems and information processing". For her thesis topic, Imane is studying the internet of things and its performance in $5 \mathrm{G}$.

Salma Rattal received her $\mathrm{PhD}$ degree in Telecommunication from Hassan II University, Casablanca, Morocco. She previously graduated as a specializing Master in Telecommunication and a network of UCD - Chouaïb Doukkali University - El Jadida. She has worked as a postdoctoral researcher at the University of Hassan II of Casablanca - Electrical Engineering Department, and at the laboratory of SNRT, Currently, she is a professor at the Moroccan School of Engineering Sciences.

Kamal Ghoumid received his PhD degree from the 'Institut Mines-Télécom, Télécom Sud-Paris', Evry, France, and 'Institute FEMTO-ST' of the Franche-Comté University (Besaçon, France), in 2008. He got his DEA (Master) 'Communication Systems' of Paris-Est University (Paris, France). He has worked as postdoctoral researcher at Jean Lamour Institute of Henri Poincaré University (Nancy, France), and at the Institut FEMTO-ST of the Franche-Comte University, Besançon. Currently, he is professor in ENSAO (Ecole Nationale des Sciences Appliquées d'Oujda) - Mohammed First University, Morocco.

Article submitted 2019-12-12. Resubmitted 2020-02-15. Final acceptance 2020-02-20. Final version published as submitted by the authors. 